\title{
Photometric measurements for visibility level computations
}

\author{
Roland Brémond PhD, E Dumont PhD, V Ledoux BEng and A Mayeur PhD \\ Laboratoire Exploitation, Perception, Simulateurs et Simulations, Laboratoire \\ Central des Ponts et Chaussees, Université Paris Est, Paris, France
}

Received 23 April 2010; Revised 2 June 2010; Accepted 10 June 2010

Visibility level is a standard quality index in road lighting design. Three photometric values are needed to compute the visibility level of a target: its luminance, the luminance of its near background and the adaptation luminance. We discuss the consequences of how these parameters are set on the ability to predict the performance of a driver at target detection. An experiment was designed using a closed-road circuit. Sixteen targets were presented twice to 34 subjects. Six visibility level assessment methods were compared. The background luminance was set as the luminance at the bottom of the target, as the mean luminance around the target, or as the luminance associated with the maximum target contrast. The adaptation luminance was set either to the background luminance or to the mean luminance. The best non-linear fit between computed visibility level and target detection performance was found using the maximum contrast over the four sides of the square target, and setting the adaptation luminance to a unique estimated road luminance instead of the local background luminance. Even so, the variability of the visibility level data suggests great caution when using it as an index of road visibility.

\section{Introduction}

A number of studies have emphasised the contribution of road lighting to road safety, ${ }^{1,2}$ with the warning that a poor lighting design may be worse than no lighting at all. ${ }^{3,4}$ Thus, road lighting may be seen as an accident countermeasure, in addition to automobile lighting.

Twenty years ago, $\operatorname{Adrian}^{5-7}$ gathered psychophysical data in order to compute the visibility of a small target on the road and proposed that the visibility level (VL) could be a reference value for road lighting design. His work followed Blackwell's definition of the $\mathrm{VL}^{8}$ as the ratio between a target

Address for correspondence: Roland Brémond, Laboratoire Central des Ponts et Chaussées, 58 Boulevard Lefebvre, 75015 Paris, France.

E-mail: roland.bremond@1cpc.fr luminance contrast $\Delta L / L_{\mathrm{b}}$ and a threshold luminance contrast $\Delta L_{\mathrm{t}} / L_{\mathrm{b}}\left(L_{\mathrm{b}}\right.$ is for the background luminance). When compared to the usual indexes such as illuminance and luminance, the VL approach is more relevant because it addresses a visual performance (target detection), which is part of the driving activity.

$\mathrm{VL}$ is proposed as a quality assessment index in the American standard 9 but not in the European standard. ${ }^{10}$ The American standard uses the small target visibility reference scenario: Road lighting should improve drivers' performance for the detection of a small target at a distance where they usually pick up information. Based on this scenario, Adrian's model can be used to compute VL values in a reference situation: A 50\% reflectance, square target of $0.18 \mathrm{~m}$ side, situated $83 \mathrm{~m}$ ahead of a 60 -year-old driver. Then, thresholds for the mean VL make it possible 
to compute a quality index from the photometric measurements of the targets and background (road surface) luminance values. In the French guidelines, the VL index is also mentioned, with a threshold set to $7,{ }^{11,12}$ however, without averaging the VL values.

Some authors have used the VL or similar visibility indexes (VI) in order to compute target visibility at the time of detection, either for road lighting ${ }^{13}$ or automobile lighting. ${ }^{14,15}$ They make the implicit hypothesis that such a VI (computed from laboratory psychophysical data) may be relevant to the visibility of targets on the road, that is, the road visibility is well enough described by the VL, even though factors such as the target shape, colour, location in the field of view, etc. are not taken into account in the model. ${ }^{12,16}$

Although a number of authors have emphasised some drawbacks of the VL model, ${ }^{17}$ in this paper, we accept the model hypotheses and focus on metrological issues. Adrian ${ }^{6}$ linked a reference driving scenario to reference psychophysical laboratory data, ${ }^{18}$ taking into account the target's angular size and contrast polarity, the driver's age, the presentation time and possible glare. The laboratory data were, however, collected in conditions quite different from a driving situation: Uniform background luminance, flat uniform luminance target, ${ }^{12}$ simple versus complex tasks ${ }^{19}$ and static versus dynamic situation. $^{20}$

In the following, we discuss an important difference between the psychophysical data and the reference driving situation: Road surfaces are not uniform in luminance. In order to compare the relevance of various measurement methods for the background luminance, an experiment was conducted on a closed-road circuit. $^{21}$ Thirty-four subjects each drove 32 laps of the circuit (half as driver and half as a passenger), and had to detect targets situated on the illuminated section of the circuit. Sixteen target positions were chosen, leading to $16 \mathrm{VL}$ values, computed for a target with an angular size of $10^{\prime}$ (which means, for a square target of $20 \mathrm{~cm}$ side, a distance of $69 \mathrm{~m}$ ). The experimental design made it possible to compare the correlation between the detection distance (DD) (dependent variable) and the VL (independent variable) computed using various methods for setting the input parameters (background and adaptation luminances). The rest of this paper addresses two issues as follows:

- How to set the background luminance and adaptation luminance so as to get the best correlation between the VL and the visibility distance? The purpose of this issue is to select, among those tested, the best photometric measurement method for VL computation, in terms of relevance to road visibility.

- Compute the VL at the moment of target detection (instead of using the conventional angular size of $10^{\prime}$ ). A given percentile of this $\mathrm{VL}_{\mathrm{d}}$ distribution ( $\mathrm{d}$ is for detection) may be used for the selection of VL threshold values in road lighting design.

\section{Material and method}

\subsection{Experimental setup}

Thirty-four adults (11 women and 23 men) with a mean age of 36 years served as subjects. None of them was familiar with the hypotheses under investigation. They were required to have owned a valid driving licence for at least 5 years. All subjects had at least $8 / 10$ corrected binocular acuity and wore the optical correction that they would normally wear while driving, if any. The subjects were randomly assigned to one of two groups; no statistical difference was observed between these two groups neither in terms of acuity nor in terms of age.

The experiment was conducted under night-time conditions on a closed road circuit, $1.2 \mathrm{~km}$ long (Figure 1). This circuit is a 


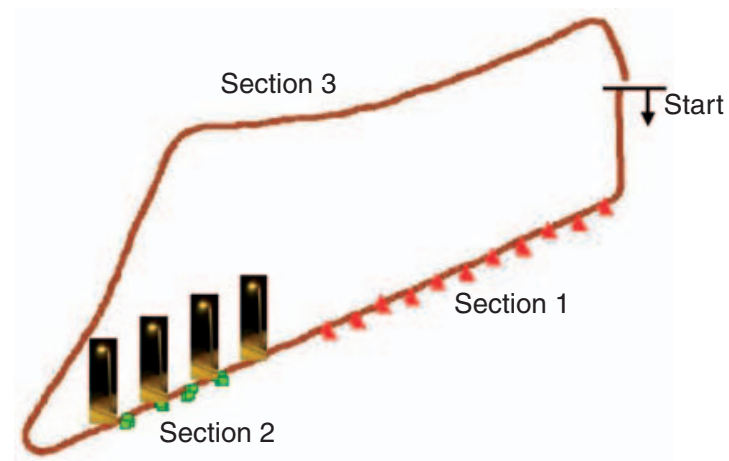

Figure 1 General shape of the closed-road circuit, with the illuminated section where the targets were set (section 2), and the previous cone section (section 1)

full-scale research facility to test roadway lighting. $^{22}$ The experiment was restricted to dry weather conditions (no rain, dry pavement). The circuit is free of road markings and was free of other vehicles during the experiment. The pavement on this section is classified as R2 in the CIE road surface classification system, ${ }^{23}$ with an average luminance coefficient (Qo) of 0.1 (R1 is for diffuse surfaces, R4 for specular surfaces).

The lighting installation was a one-side arrangement of four aluminium poles, with adjustable spacing. A $45 \mathrm{~m}$ spacing was chosen, and the mounting height of the luminaires was adjusted to $8 \mathrm{~m}$. Each luminaire contained a $150 \mathrm{~W}$ high-pressure sodium lamp provided by Thorn Lighting. Light output was controlled by potentiometers, which allowed the light power to be modulated down to $50 \%$ of the nominal value. This design was not intended to mimic a high quality road lighting installation but to produce both dark and light areas on the roadway. One $20-\mathrm{cm}$-side square target with a $60 \%$ reflectance was used. The luminance contrast was modified by physically displacing the target on the non-uniformly illuminated pavement. In the portion of the circuit equipped with road lighting, a small white point was painted every metre on the two sides of the road, allowing the experimenters to position the target accurately.

Eleven pairs of traffic cones were positioned on the first section of the circuit (Section 1 in Figure 1). The gap between the cones $(4 \mathrm{~m})$ was wide enough for the drivers to pass through at around $30 \mathrm{~km} /$ hour. Such traffic cones had already been used in a field experiment by Van Bommel and Tekelenburg $^{3}$ in order to increase the workload. The drivers were instructed to pass between the cones in the first part of the circuit and to drive straight in the second part. The first target position was $77 \mathrm{~m}$ after the last cone. The target positions were selected in order to get a large range of DD; they were situated on one of two lines, either on the right or on the left side of the road.

The speed and the position along the circuit were measured using a Global Positioning System in the vehicle. The participants were asked to press a button as soon as they had detected a target. The distance between the vehicle and the target at that moment was considered as the DD. Low-beam headlights were used at all times to ensure safety and to match reality.

Subjects were split into two groups (A and B) and completed 32 laps in two phases (1 and 2). Phases 1 and 2 were counterbalanced within groups (A and B). Two status conditions were defined as follows: passenger (P) and driver (D). For the driver, the button to be pressed was located on the steering wheel. For the passenger, the same subjects had to detect the target stimulus while the experimenter was driving. ${ }^{21}$ In the rest of this paper, we focus on the link between a visual performance (target detection) and VL.

\subsection{Photometric measurements}

\subsubsection{Luminance measurements}

Photometric measurements were performed with a Pritchard 1980A photometer in order to compute the target contrast and 
the VL for a standard observer. The measurements were made from a distance of $30 \mathrm{~m}$ and at a height of $1.20 \mathrm{~m}$ above the road surface. For each target location, five points were measured: one on the target and four on the road surface (top, bottom, left and right of the target), with a $2^{\prime}$ aperture.

All measurements were performed without taking the vehicle headlights into account. Supplementary illuminance measurements were made in order to check for changes in target contrast and $\mathrm{VL}$ in the presence of the headlamps, which were tuned to low power, and dipped as low as possible. These measurements showed that the changes in contrast did not alter the target visibility for distances greater than $35 \mathrm{~m}$; for smaller distances, depending on the target position, the headlights may have impacted the luminance contrast.

\subsubsection{VL computation}

From the target and background luminance values, the VL values were computed using Adrian's model, for a 25-year-old subject, 0.2 seconds of observation time and $10^{\prime}$ of angular size as in the original paper. ${ }^{6}$ Three methods were tested for setting the background luminance: Using the luminance of the pavement at the bottom of the target $(\operatorname{method} \mathrm{A})$, the mean luminance around the target (mean of the four points: top, bottom, left and right, method B) and the luminance that leads to the maximum contrast for the target (method C). Table 1 shows the background luminance values computed for the 16 target locations for methods A, B and C. The luminances are quite low because the lighting installation was designed to produce target DDs between $30 \mathrm{~m}$ and $200 \mathrm{~m}$. Note that the differences between the luminance contrast values are due to the fact that the illumination of the pavement is not homogeneous, whereas in Adrian's model, the 'background' luminance is assumed to be uniform.
Table 1 Background luminances for the 16 target locations obtained using various methods (in $\mathrm{cd} / \mathrm{m}^{2}$ )

\begin{tabular}{lllll}
\hline $\begin{array}{l}\text { Target } \\
\text { location }\end{array}$ & $\begin{array}{l}\text { Method } \\
\mathrm{A}\end{array}$ & $\begin{array}{l}\text { Method } \\
\mathrm{B}\end{array}$ & $\begin{array}{l}\text { Method } \\
\mathrm{C}\end{array}$ & $\begin{array}{l}\text { (Max-Min)/ } \\
\text { Max (\%) }\end{array}$ \\
\hline 1 & 0.19 & 0.27 & 0.19 & 29.0 \\
2 & 0.85 & 0.77 & 0.51 & 40.0 \\
3 & 0.24 & 0.28 & 0.24 & 15.0 \\
4 & 0.32 & 0.32 & 0.58 & 44.4 \\
5 & 0.30 & 0.27 & 0.21 & 30.0 \\
6 & 0.38 & 0.35 & 0.28 & 26.3 \\
7 & 0.58 & 0.48 & 0.43 & 25.9 \\
8 & 0.60 & 0.55 & 0.37 & 38.3 \\
9 & 0.07 & 0.37 & 0.07 & 80.9 \\
10 & 0.40 & 0.35 & 0.28 & 30.0 \\
11 & 0.12 & 0.24 & 0.12 & 48.9 \\
12 & 0.65 & 0.53 & 0.46 & 29.2 \\
13 & 0.30 & 0.27 & 0.22 & 26.7 \\
14 & 0.25 & 0.33 & 0.25 & 23.1 \\
15 & 0.19 & 0.28 & 0.19 & 32.1 \\
16 & 0.29 & 0.27 & 0.23 & 20.7 \\
\hline
\end{tabular}

In addition, we compared two methods for setting the adaptation luminance. The first method was to compute the detection threshold $\Delta L_{\mathrm{t}}$ using the target background luminance as usual (this value depends on the measurement methods $\mathrm{A}, \mathrm{B}$ or $\mathrm{C}$, see the luminance values in Table 1). This leads to three VL computation methods (M1, M2 and M3 in Table 2). The second method was to estimate the adaptation luminance as the mean background luminance over the 16 target locations (mean of the $16 \times 4$ luminance measurements on the road surface). This leads to methods M4, M5 and M6 in Table 2. The rationale of this second method was to set the adaptation luminance to a unique value $\left(L_{\mathrm{a}}=0.40 \mathrm{~cd} / \mathrm{m}^{2}\right)$ for all targets, even if the adaptation value is known to be difficult to estimate.

The first striking result, prior to any comparison with performance data, is the very strong dependence of VL on the measurement method and on the adaptation luminance estimation method. The deviation between methods, given by

$$
\Delta=(\mathrm{VL} \max -\mathrm{VL} \min ) / \mathrm{VL} \max ,
$$

would have always been zero with a uniform background. The last column of Table 2 
Table 2 VL values computed following Adrian's method, depending on the method to set the photometric parameters

\begin{tabular}{|c|c|c|c|c|c|c|c|}
\hline \multirow[t]{2}{*}{ Target } & \multicolumn{3}{|l|}{$L_{\mathrm{a}}=L_{\mathrm{b}}$} & \multicolumn{3}{|l|}{$L_{\mathrm{a}}=\langle L>$} & \multirow{2}{*}{$\begin{array}{c}(\operatorname{Max}-\operatorname{Min}) / \\
\operatorname{Max}(\%)\end{array}$} \\
\hline & $\begin{array}{l}\text { Bottom } \\
\text { M1 }\end{array}$ & $\begin{array}{l}\text { Mean } \\
\text { M2 }\end{array}$ & $\begin{array}{l}\text { Max } \\
\text { M3 }\end{array}$ & $\begin{array}{l}\text { Bottom } \\
\text { M4 }\end{array}$ & $\begin{array}{l}\text { Mean } \\
\text { M5 }\end{array}$ & $\begin{array}{l}\text { Max } \\
\text { M6 }\end{array}$ & \\
\hline 1 & 6.39 & 3.32 & 6.39 & 7.79 & 4.82 & 7.79 & 57.4 \\
\hline 2 & 14.91 & 16.98 & 25.98 & 40.81 & 43.77 & 53.42 & 72.1 \\
\hline 3 & 0.81 & 0.43 & 0.81 & 1.11 & 0.22 & 1.11 & 80.2 \\
\hline 4 & 1.61 & 1.61 & 8.50 & 0.87 & 0.87 & 6.49 & 89.8 \\
\hline 5 & 8.46 & 9.70 & 12.75 & 12.98 & 14.10 & 16.32 & 48.2 \\
\hline 6 & 2.13 & 2.89 & 5.01 & 3.71 & 4.82 & 7.42 & 71.3 \\
\hline 7 & 10.55 & 13.64 & 15.51 & 23.37 & 27.08 & 28.94 & 63.5 \\
\hline 8 & 3.45 & 4.49 & 9.50 & 7.79 & 9.65 & 16.32 & 78.9 \\
\hline 9 & 10.75 & 2.59 & 10.75 & 8.53 & 1.51 & 8.53 & 85.9 \\
\hline 10 & 4.14 & 5.57 & 8.02 & 7.42 & 9.27 & 11.87 & 65.1 \\
\hline 11 & 13.15 & 6.24 & 13.15 & 12.98 & 8.53 & 12.98 & 52.5 \\
\hline 12 & 2.23 & 4.59 & 6.32 & 5.19 & 9.65 & 12.24 & 81.8 \\
\hline 13 & 3.38 & 4.34 & 6.23 & 5.19 & 6.31 & 8.16 & 58.6 \\
\hline 14 & 1.86 & 0.40 & 1.86 & 2.60 & 0.22 & 2.60 & 91.5 \\
\hline 15 & 4.56 & 1.50 & 4.56 & 5.56 & 2.23 & 5.56 & 73.0 \\
\hline 16 & 1.23 & 1.79 & 3.05 & 1.85 & 2.60 & 4.08 & 69.8 \\
\hline Mean & 5.60 & 5.01 & 8.65 & 9.23 & 9.10 & 12.74 & 71.2 \\
\hline
\end{tabular}

shows that the deviation ranges between $48 \%$ and $91 \%$, depending on the target position, showing that these choices are of great importance for practical applications. Even if the lighting installation was not representative of illumination standards, this raises some questions.

As the various methods lead to various mean VL values (last row of Table 2), one may argue that the deviation between methods may be due to a scale factor. This idea was tested by normalising the results of each method (M1-M6) so that all methods lead to the same mean VL over the 16 target positions. After correction by such a scale factor for each method, the average deviation drops from $71 \%$ to $58 \%$, which is still high.

\section{Results}

The statistical effects of the independent variables and co-variables in this experiment are analysed in more detail elsewhere using an ANOVA. ${ }^{21}$ The main points are the practice effect (performance is higher in the second phase than in the first phase, with a
Table 3 Mean DD and SD (in metres) for each target position

\begin{tabular}{lrll}
\hline Target & Mean & SD & $\begin{array}{l}\text { SD/Mean } \\
(\%)\end{array}$ \\
\hline 1 & 58.79 & 28.14 & 47.9 \\
2 & 180.38 & 78.31 & 43.4 \\
3 & 62.50 & 33.61 & 53.8 \\
4 & 104.65 & 46.17 & 44.1 \\
5 & 160.54 & 61.34 & 38.2 \\
6 & 122.09 & 50.18 & 41.1 \\
7 & 191.36 & 67.51 & 35.3 \\
8 & 130.26 & 52.48 & 40.3 \\
9 & 158.94 & 54.99 & 34.6 \\
10 & 178.77 & 61.46 & 34.4 \\
11 & 70.44 & 31.49 & 44.7 \\
12 & 140.13 & 39.48 & 28.2 \\
13 & 149.11 & 51.91 & 34.8 \\
14 & 71.45 & 44.25 & 61.9 \\
15 & 81.72 & 27.67 & 33.9 \\
16 & 85.11 & 36.62 & 43.0 \\
\hline
\end{tabular}

The mean DD values are computed over 68 detections (34 subjects $\times 2$ trials). The $\mathrm{SD} /$ mean ratio gives an index of the variation of the visual performance across subjects.

statistically significant difference) and the status effect (subjects perform better as passengers than as drivers). A statistically significant influence of the VL was found $(F(15$, $465)=29.12, p<0.0001)$ with a medium effect size, $\omega^{2}=0.13$.

\subsection{Effect of measurement method}

Table 3 shows the mean DD for each target location. We have compared the ability of the $\mathrm{VL}$ to predict the target detection performance, using the six VL computation methods (M1-M6) proposed in Section 2. The justification for this comparison was that using the VL for road lighting design makes the implicit assumption that VL is an index of visual performance (target detection) while driving.

The comparison was first made by assessing a linear relation between the VL and the DD of a target, the latter being taken as an index of driving performance. Then, a nonlinear increasing relation was tested. The Pearson correlation coefficient $(r)$ rates the 
Table 4 Correlation of the DD against VL for various methods to select the input parameters for VL computation

\begin{tabular}{|c|c|c|c|c|c|c|c|}
\hline \multicolumn{2}{|c|}{ Correlation coefficient } & \multirow{2}{*}{$\frac{\mathrm{M} 1}{0.406}$} & \multirow{2}{*}{$\frac{\mathrm{M} 2}{0.699}$} & \multirow{2}{*}{$\frac{\text { M3 }}{0.630}$} & \multirow{2}{*}{$\frac{\mathrm{M} 4}{0.563}$} & \multirow{2}{*}{$\frac{\text { M5 }}{0.644}$} & \multirow{2}{*}{$\frac{\mathrm{M} 6}{0.634}$} \\
\hline Pearson & $r$ & & & & & & \\
\hline & $r_{2}$ & 0.397 & 0.704 & 0.648 & 0.564 & 0.651 & 0.646 \\
\hline Spearman & $\rho$ & 0.435 & 0.674 & 0.606 & 0.506 & 0.669 & 0.706 \\
\hline & $\rho_{2}$ & 0.509 & 0.750 & 0.676 & 0.576 & 0.728 & 0.764 \\
\hline
\end{tabular}

The Pearson correlation coefficient quantifies the linearity of the relation between DD and VL, whereas the Spearman correlation coefficient quantifies a possible increasing function for the relation between DD and VL.

extent to which the relation between the DD and the VL is linear. Table 4 shows $r$ values computed with our detection performance data, depending on the methods (M1-M6) for computing the VL. In order to control the robustness of these results, we also computed the $r$-value ( $r_{2}$ in Table 4) based on the median of the DD instead of the mean. One advantage of the median is that it does not depend on extreme values, such as detection at distances lower than $35 \mathrm{~m}$ where the vehicle headlamps may have biased the performance. From Table 4, methods M2 and M5 lead to the highest values for both $r$ and $r_{2}$, whatever the method to compute the distance. Recall that both M2 and M5 use the 'mean of four points' method to compute the background luminance, and only differ in the choice of the adaptation luminance. The results also suggest that M2 gives better results than M5. That is, using the 'mean of four points' measurement method for the background luminance, the adaptation luminance had better be set to the local background luminance, rather than to a unique luminance value for the whole road section.

Although these results are in favour of a 'four points' computation of the background luminance for VL computations, the linear hypothesis is not strongly supported by our data: even the best method leads to $r=0.699$ $\left(r_{2}=0.704\right)$, which means that $48.9 \%$ of the variance is explained by the linear model. Looking at Figure 2, it appears that a nonlinear model is more relevant given the shape of the data. Thus, a non-linear increasing relation was tested with the Spearman

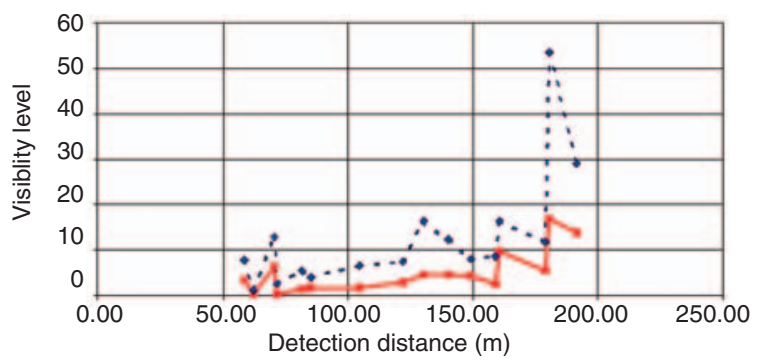

Figure 2 Visibility level, computed with M2 (solid) and M6 (dotted) methods, as a function of the mean DD

correlation coefficient $\rho$, which uses the ranks instead of the actual values. Table 4 also shows the results of this analysis ( $\rho_{2}$ is for the Spearman coefficient computed with the median DD). The results are quite different in terms of the preferred method: the M6 method gives the best results.

As the DD does not result from the photometric inputs only, the VL cannot fully explain the detection performance. ${ }^{19-21}$ Still, Table 4 shows that photometric inputs have a strong impact on the DD $\left(\rho_{2}=0.764\right.$ for M6). Using Student $t$ tests, the hypothesis that $\rho_{2}=0$ is rejected for all methods with $p=0.05$; it is rejected for M2, M3, M5 and M6 for $p=0.01$ and for M2 and M6 for $p=0.001$. However, the confidence intervals range from $[0.02,0.80]$ for $\mathrm{M} 1$ to $[0.43,0.91]$ for M6, showing a common range $[0.43,0.80]$ for all six methods. This means that, unfortunately, although the computed Spearman and Pearson correlation coefficients are different, these differences are not statistically significant. This result was confirmed by the 
Cochran-Hotelling test, with $p$-values always above 0.13 . This result is consistent with the statement that the DD cannot easily be predicted based on photometric measurements alone. The only recommendation one can propose at this stage is based on a rule of thumb: We prefer a VI built on a method, which leads to the highest Spearman correlation coefficient, given the available data; moreover, choosing M6 leads to the smallest $p$-value $(p=0.00057)$ when testing the hypothesis that $\rho_{2}=0$. Thus, we recommend at this stage the M6 method for VL computation, with the background luminance set to the value that maximises the target contrast, and adaptation luminance set to a unique luminance value, representative of the road illumination, rather than a local background luminance. More data is needed in order to discriminate between the proposed methods on a statistical basis.

Several biases may be responsible for the unexplained variance. First, the DD was slightly underestimated in our methodology because the reaction time between the visual detection and the motor response (pushing a button) was not taken into account. Given the mean speed at the moment of target detection, the order of magnitude of this bias is $5 \mathrm{~m}$. More important, the luminance measurements were performed with a unique geometry $(30 \mathrm{~m}$ from the target, $1.20 \mathrm{~m}$ high), while this geometry obviously varies with the distance to target. Thus, the background luminance measurements are not set to the true background luminance at the moment of detection.

However, a question remains: Is the VL computed for a $10^{\prime}$ target a good index of the target detection performance on the road? And if not, what could be the alternative? Answering these questions is beyond the scope of this paper; however, looking at individual performance may give some insights. Table 3 shows the mean and standard deviation (SD) of the DDs for the
16 target positions. The high values of the ratio mean/SD serves to emphasise, again, the idea that a true predictor of the individual performance cannot be based on photometric data alone.

\subsection{VL at the time of target detection}

In the previous section, the VL was computed following Adrian's method, assuming that the angular size of the target was $10^{\prime}$. This hypothesis is suited for a measurement scenario but does not correspond to the actual angular size of the target at the moment of detection. Our data, however, also allows us to compute this angular size for each target detection, leading to the actual VL at the moment of target detection. In the following, the VL at the moment of detection is denoted as $\mathrm{VL}_{\mathrm{d}}$ ( $\mathrm{d}$ is for detection).

These data are interesting for several reasons. First, Gallagher and Meguire used a percentile of $\mathrm{VL}_{\mathrm{d}}$ as a criterion, arguing that roadway lighting should allow $85 \%$ (or $95 \%$ ) of the drivers to detect the targets at a safe distance. ${ }^{13}$ Based on our data, this leads to various VL thresholds, depending on the computational method. These thresholds are the field factors to be applied to laboratory thresholds for road lighting applications. Table 5 shows three percentiles of $\mathrm{VL}_{\mathrm{d}}$ $(85 \%, 95 \%$ and $99 \%)$ for the six methods M1-M6.

Direct comparisons with the data from Gallagher and Meguire is difficult because their percentiles were computed from a VI that is different from Blackwell's VL. However, partial data allows us to infer a corresponding value for VL of 8.3 for the 95 th percentile at $40 \mathrm{~km} /$ hour, which was the average speed in our experiment. This value may be compared to $\mathrm{VL}=9.4$ for method M2 and $\mathrm{VL}=9.2$ for method M6.

Another interesting issue that may be tested with the $\mathrm{VL}_{\mathrm{d}}$ data is the following. One may argue that the previous comparison between methods M1-M6 is unfair, as the 
Table 5 Field factor values estimated using percentile data from the $\mathrm{VL}_{\mathrm{d}}$ values

\begin{tabular}{lrrrrrr}
\hline Criterion & M1 & M2 & M3 & M4 & M5 & M6 \\
\hline $85 \%$ & 6.6 & 4.9 & 8.8 & 3.5 & 3.5 & 5.1 \\
$95 \%$ & 15.2 & 9.4 & 18.5 & 7.4 & 5.6 & 9.2 \\
$99 \%$ & 21.7 & 13.9 & 24.8 & 12.4 & 13.2 & 19.3 \\
\hline
\end{tabular}

Table $6 \mathrm{SD}$ of the $\mathrm{VL}_{\mathrm{d}}$ values for various methods to select the input parameters for VL computation

\begin{tabular}{lllllll}
\hline & M1 & M2 & M3 & M4 & M5 & M6 \\
\hline Mean VL & 3.25 & 2.39 & 4.61 & 1.86 & 1.67 & 2.66 \\
VL $_{d}$ SD & 3.62 & 1.91 & 3.41 & 1.70 & 1.53 & 1.87 \\
SD/Mean & 1.11 & 0.80 & 0.74 & 0.91 & 0.91 & 0.70 \\
\hline
\end{tabular}

DDs lower than $35 \mathrm{~m}$ were removed from the data.

computed VL did not take into account the true target angular size at the time when it was detected (a conventional value of $10^{\prime}$ was preferred). Even if it is irrelevant for road lighting quality assessment, one may check if the previously preferred method still appears relevant with the $\mathrm{VL}_{\mathrm{d}}$ data. The answer to this question is not straightforward because the VL hypothesis would, ideally, state that the $\mathrm{VL}_{\mathrm{d}}$ should be the same at the moment of target detection, whatever the target size, luminance and contrast. Of course, this is not what we found in our data, and one way to test the relevance of a given computation method is to rate the $\mathrm{SD}$ of $\mathrm{VL}_{\mathrm{d}}$ over the mean $\mathrm{VL}_{\mathrm{d}}$. This is shown in Table 6 .

Rating the method consistency by the SD/ mean ratio of the $\mathrm{VL}_{\mathrm{d}}$ values, the M6 method is, once again, first among the six proposed methods. However, the most interesting (and worrying) results are the very high $\mathrm{SD}$ values with respect to the mean $\mathrm{VL}_{\mathrm{d}}$. It suggests that care should be taken whenever using the VL as an index of target detection on the road.

\section{Conclusions}

Evidence has been given, using a field experiment, that the photometric measurement method used strongly impacts the VL value. Moreover, it has been shown that some methods are more relevant than others, in the sense that they lead to a better fit, based on the Spearman correlation coefficient, between the VL and a measure of visual performance, the target DD. Our data suggest that the 'maximum contrast' method should be preferred (leading to a monotonic, non-linear relation between VL and DD), and that the adaptation luminance should be set to the same value for all targets rather than to the local background luminance values. However, these conclusions were not supported by a statistical comparison between the six tested methods as the available data lead to quite large confidence intervals about the correlation coefficients. Thus, our recommendations are subject to reevaluation with more data.

Finally, the experimental data suggest that VL should be used with caution when looking for a prediction of a target detection performance, as the variability in the performance has the same order of magnitude as the performance itself. New experiments may still be useful, using an onboard video photometer in order to be able to compute VLs with luminances taken from the point of view of the driver at the moment of target detection, to see if these new data improve the quality of the relation between VL and DD.

Field experiments have been conducted to extend findings about visual performance under mesopic light levels to a driving context. Very few studies have focused on the VL in a dynamic driving situation. Thirty-five years ago, Gallagher and Meguire ${ }^{13}$ explored the empirical relationship between a measure of driver visual performance under road lighting (time to target) and several methods of quantifying the visibility. They analysed the field measurements of visual performance of 941 drivers and found $\mathrm{VL}_{\mathrm{d}}$ values between 4 and 12 at the moment of detection. The visibility of a pedestrian dummy and of discs of varying size and contrasts were also 
measured under dynamic night driving conditions by Hills. ${ }^{24}$ The three subjects of the study were driving at $32 \mathrm{~km} /$ hour. They had to press a button when the target was just visible and gave a verbal answer about the nature of the object. Hills found that a VL of 4 was required as a 'just visible criterion'. More recently, Ising et al. ${ }^{14,15}$ used experimental data from Olson et $a l^{25}$ to compute $\mathrm{VL}_{\mathrm{d}}$ values with Adrian's model but without road lighting. They found that with headlights only, the 50th percentile $\mathrm{VL}_{\mathrm{d}}$ ranges between 1 and 23 . The rationale for these studies was to recommend a threshold value for the VL (or mean VL in the IESNA standard'), taken as a 'field factor'. Our results are in good agreement with the Gallagher and Meguire data. However, they emphasise the fact that the field factor value depends on the measurement method.

\section{Acknowledgements}

The authors would like to express appreciation to Damien Lesbats and Christophe Poivert (CETE-NC) for their help in the experimental setting and sessions, and to Ariane Tom and Jean-Philippe Tarel for helpful advice. The authors are grateful for the subject's participation. This study was partially supported by a grant from the Laboratoire Central des Ponts et Chaussées.

\section{References}

1 Commission Internationale de l'Eclairage. Road Lighting as an Accident Countermeasure, CIE Publication 93. Vienna: CIE, 1992.

2 Wanvik PO. Effects of road lighting: an analysis based on Dutch accident statistics 1987-2006. Accident Analysis and Prevention 2009; 41: 123-128.

3 Van Bommel JM, Tekelenburg J. Visibility research for road lighting based on a dynamic situation. Lighting Research and Technology 1986; 18: 37-39.
4 Mace DJ, Porter RJ. Fixed roadway lighting: the effect of lighting geometry and photometry on target visibility and driver comfort: Proceedings of the 83rd Transportation Research Board Annual Meeting, Washington: TRB, 2004.

5 Adrian W. Visibility levels under night-time driving conditions. Journal of the Illuminating Engineering Society 1987; 16: 3-12.

6 Adrian W. Visibility of targets: model for calculation. Lighting Research and Technology 1989; 21: 181-188.

7 Adrian W. Fundamentals of roadway lighting. Light and Engineering 2004; 12: 57-71.

8 Commission Internationale de l'Eclairage. An Analytic Model for Describing the Influence of Lighting Parameters Upon Visual

Performance, CIE Publication 19/2. Vienna:

CIE, 1981.

9 Illuminating Engineering Society of North America. American National Standard Practice for Roadway Lighting, IESNA Publication RP8-00. New York: IESNA, 2000.

10 European Committee for Standardization (CEN). EN 13201 Series: Road Lighting. Brussels: CEN, 2004-2005.

11 Lecocq J. Visibility level in outdoor lighting. Adrian model applied to spherical cap targets: Proceedings of the 22nd Session of the CIE, Melbourne, Australia. Vienna: CIE, 1991.

12 Lecocq J. Calculation of the visibility level of spherical targets in roads. Lighting Research and Technology 1999; 31: 171-175.

13 Gallagher VP, Meguire PG. Driver Visual Needs in Night Driving. Transportation Research Board Special Report 156. Washington: TRB, 1975.

14 Ising KW. Threshold visibility levels required for night-time pedestrian detection in a modified Adrian/CIE visibility model. Leukos 2008; 5: 63-75.

15 Ising KW, Fricker TRC, Lawrence JM, Siegmund GP. Threshold Visibility Levels for the Adrian Visibility Model Under Night-Time Driving Conditions. Warrendale, PA: Society of Automotive Engineers, 2003.

16 Akashi Y, Rea MS, Bullough JD. Driver decision making in response to peripheral moving targets under mesopic light levels. Lighting Research and Technology 2007; 39: 53-67. 
17 Brémond R. Quality indexes for road lighting: a review: Proceedings of the 26th Session of the CIE, Beijing, China. Vienna: CIE, 2007.

18 Blackwell HR. Contrast thresholds of the human eye. Journal of the Optical Society of America 1946; 36: 624-643.

19 Mayeur A, Brémond R, Bastien C. The effect of task and eccentricity of the target on detection thresholds in mesopic vision: Implications for road lighting. Human Factors 2008; 50: 712-721.

20 Mayeur A, Brémond R, Bastien C. Effects of the viewing context on peripheral target detection: Implications for road lighting design. Applied Ergonomics 2010; 41: 461-468.

21 Mayeur A, Brémond R, Bastien C. The effect of the driving activity on target detection as a function of the visibility level. Transportation
Research Part F, Traffic Psychology and Behaviour 2010; 13: 115-128.

22 Ménard J, Cariou J. Road lighting: assessment of an installation based on the contrast of a standard target. Lighting Research and Technology 1994; 26: 19-22.

23 Commission Internationale de l'Eclairage. Road Surfaces and Lighting, CIE Publication 66. Vienna: CIE, 1984.

24 Hills BL. Visibility under night driving conditions. Part 2. Field measurements using disc obstacles and a pedestrian dummy. Lighting Research and Technology 1975; 7: 251-258.

25 Olson PL, Aoki T, Battle DS, Flannagan MJ. Development of a Headlight System Performance Evaluation Tool. Ann Arbor, MI: The University of Michigan Transportation Research Institute, 1990. 